\title{
An adaptive numerical scheme for a partial integro-differential equation
}

F. Georgiou ${ }^{1} \quad$ B. P. Lamichhane ${ }^{2} \quad$ N. Thamwattana ${ }^{3}$

(Received 28 February 2019; revised 26 September 2019)

\begin{abstract}
One method of modelling cell-cell adhesion gives rise to a partial integro-differential equation. While non-adaptive techniques work in the numerical modelling of such an equation, there are also many opportunities for optimisation. The studied partial integro-differential equation has a tendency to produce aggregations leaving large regions where both the function value and derivative are equal to zero, leading to a higher resolution than needed and lower than desired resolution where the aggregations form. In order to overcome this we develop an adaptive scheme in both space and time using a modified form of Matlab's ode45 and finite volume methods to more efficiently simulate the studied partial integro-differential equation. We use our numerical scheme to simulate the problem presented by Armstrong et al. [J. Theor. Biol. 243 (2006), pp. 98-113] and compare results.
\end{abstract}

Dor:10.21914/anziamj.v60i0.14066 gives this article, (c) Austral. Mathematical Soc. 2019. Published October 9, 2019, as part of the Proceedings of the 18th Biennial Computational Techniques and Applications Conference. ISSN 1445-8810. (Print two pages per sheet of paper.) Copies of this article must not be made otherwise available on the internet; instead link directly to the DOI for this article. 


\section{Contents}

1 Introduction

C188

1.1 Cell-cell adhesion . . . . . . . . . . . . . . . . . C188

2 Numerical scheme

C189

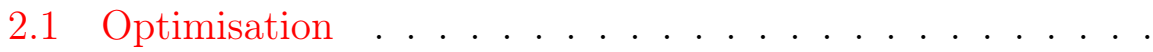

3 Results

C192

4 Conclusion

C198

\section{Introduction}

\subsection{Cell-cell adhesion}

Cell-cell adhesion is a biological process by which cells bind or stick to each other through transmembrane proteins called cell-adhesion molecules. It is through this process that tissue is formed, maintained and eventually broken down. In 1955, Townes and Holtfretter [8] showed using amphibian cells that cell sorting is dependant on cell adhesion. They found that dissociated amphibian cells return to their original configuration once the $\mathrm{pH}$ of their solution is returned to normal. Steinburg $[6,5,7]$ later found that cell envelopment is transitive; that is, if given three cell types $\mathrm{A}, \mathrm{B}$ and $\mathrm{C}$, if A envelopes B and B envelopes C then A envelopes C.

In 2006, Armstrong et al. [1] proposed a continuum approach to modelling cell-cell adhesion and developed a non-local advection model that was able to replicate Steinberg's experiments $[6,5,7]$. Cells were modelled using a conservative system where cells only undergo two types of movement: random diffusion and directed adhesion. For the adhesive movement, each cell is assumed to have a sensing radius $R$ which represents the range over 
which cells can detect their surroundings. This sensing radius may be larger than the average cell radius through the extension of cell protrusions or mechanosensing. Sheratt et al. [4] extended the model of Armstrong et al. [1] to include generalised cell kinetics (i.e., cell birth and death). For $\mathrm{N}$ cell types, the $\mathrm{jth}$ cell type is modelled by

$$
\begin{aligned}
\frac{\partial u_{j}}{\partial t} & =D_{j} \frac{\partial^{2} u_{j}}{\partial x^{2}}-\frac{\partial}{\partial x}\left[u_{j} K_{j}(\underline{u})\right]+c_{j}(\underline{u}), \\
K_{j}(\underline{u}) & =\frac{\phi_{j}}{R_{j}} \int_{-R_{j}}^{R_{j}} \sum_{k=1}^{N} S_{j k} g_{j k}\left[u_{j}\left(x+x_{0}\right), u_{k}\left(x+x_{0}\right)\right] \omega_{j}\left(x_{0}\right) d x_{0},
\end{aligned}
$$

where $\mathfrak{u}_{j}(x, t)$ is the density of cell type $\mathfrak{j}, \underline{u}$ is a vector of cell densities with $j$ th element $u_{j}(x, t), D_{j}$ is the diffusion coefficient of cell type $j, c_{j}$ is a function representing the $j$ th cell kinetics, $R_{j}$ is the sensing radius of the $j$ th cell type, $\phi_{j}$ is a constant related to viscosity, $S_{j k}$ is the adhesion strength between cell types $j$ and $k, g_{j k}\left[u_{j}\left(x+x_{0}\right), u_{k}\left(x+x_{0}\right)\right]$ describes the forces and their dependence on local cell densities, and $\omega_{j}\left(x_{0}\right)$ describes how the direction and magnitude of the force change with position $x_{0}$.

We consider $g_{j k}$ of a logistic form, $\omega_{j}\left(x_{0}\right)=\operatorname{sign}\left(x_{0}\right), R_{j}=\phi_{j}=1$, periodic boundary conditions (i.e., $u_{j}(t, 0)=u_{j}(t, L)$ for domain length $L$ ), and a static cell population (i.e., $\boldsymbol{c}_{\mathfrak{j}}(\underline{\mathrm{u}})=0$ ).

\section{Numerical scheme}

We now derive a numerical scheme for a single population modelled by

$$
\frac{\partial u}{\partial t}=D \frac{\partial^{2} u}{\partial x^{2}}-\frac{\partial}{\partial x}[u K(u)]
$$

where

$$
K(u)=S_{u u} \int_{-1}^{1} \max [u(1-u), 0] \operatorname{sign}\left(x_{0}\right) d x_{0} .
$$


Table 1: Symbol definitions in relation to an arbitrary grid cell $i$. Symbol Definition

$\Delta x_{i} \quad$ the spatial width of the grid cell $i$ in the $x$ direction

$h_{i+\frac{1}{2}}$

$x_{i}$

$u_{i} \quad$ the average density of the population $u$ over the grid cell $i$

Table 1 defines terms used in the numerical scheme for arbitrary grid cell $i$. For the diffusion term we use a standard first order central differencing scheme, thus using the terms defined in Table 1 ,

$$
\frac{\partial^{2} \mathrm{u}}{\partial x^{2}} \approx \frac{1}{\Delta x_{i}}\left(\frac{\mathrm{u}_{i+1}-\mathrm{U}_{\mathrm{i}}}{\mathrm{h}_{\mathrm{i}+\frac{1}{2}}}-\frac{\mathrm{u}_{\mathrm{i}}-\mathrm{U}_{\mathrm{i}-1}}{\mathrm{~h}_{\mathrm{i}-\frac{1}{2}}}\right) .
$$

We leave the advection term in conservative flux form and use an upwinding scheme with a linear Riemann approximator [2]. To evaluate the integral K(u) we use a rectangle method (or midpoint rule) and sum the volumes of each grid cell within the sensing radius $R$. This gives for our integral

$$
\begin{aligned}
\mathrm{K}\left[\mathrm{u}\left(\mathrm{x}_{\mathrm{i}}\right)\right] \approx \mathrm{S}_{\mathrm{uu}} & \left(\sum_{k=1}^{n} \Delta \mathrm{x}_{\mathrm{i}+\mathrm{k}} \max \left[\mathrm{u}_{\mathrm{i}+\mathrm{k}}\left(1-\mathrm{u}_{\mathrm{i}+\mathrm{k}}\right), 0\right]\right. \\
& \left.-\sum_{\mathrm{k}=1}^{\mathrm{m}} \Delta \mathrm{x}_{\mathrm{i}-\mathrm{k}} \max \left[\mathrm{u}_{i-\mathrm{k}}\left(1-\mathrm{U}_{\mathrm{i}-\mathrm{k}}\right), 0\right]\right),
\end{aligned}
$$

where $\mathrm{n}$ and $\mathrm{m}$ are selected such that

$$
\sum_{k=1}^{n} \Delta x_{i+k}=R-\frac{\Delta x_{i}}{2} \text { and } \sum_{k=1}^{m} \Delta x_{i-k}=R-\frac{\Delta x_{i}}{2} .
$$

Next, let $F_{i}=U_{i} K\left(U_{i}\right)$ and then using the Riemann approximator we find that

$$
\mathrm{W}_{\mathrm{i}-\frac{1}{2}}=\mathrm{U}_{\mathrm{i}}-\mathrm{U}_{\mathrm{i}-1}
$$


and

$$
S_{i-\frac{1}{2}}= \begin{cases}\left(F_{i}-F_{i-1}\right) W_{i-\frac{1}{2}}^{-1}, & W_{i-\frac{1}{2}} \neq 0, \\ \left(F_{i}-F_{i-1}\right) h_{i-\frac{1}{2}}^{-1}, & W_{i-\frac{1}{2}}=0 .\end{cases}
$$

Finally we define $F_{i-\frac{1}{2}}=W_{i-\frac{1}{2}} S_{i-\frac{1}{2}}$, and then the advection component becomes

$$
\frac{\partial}{\partial x}\left[u_{i} K(\underline{u})\right] \approx \frac{1}{\Delta x_{i}}\left[F_{i-\frac{1}{2}} \mathcal{H}\left(S_{i-\frac{1}{2}}\right)+F_{i+\frac{1}{2}} \mathcal{H}\left(-S_{i+\frac{1}{2}}\right)\right],
$$

where $\mathcal{H}$ is the Heaviside function defined by

$$
\mathcal{H}(x)= \begin{cases}0, & x<0 \\ 1, & x \geq 0\end{cases}
$$

For our time step we use the Dormand-Prince method, which is an explicit adaptive Runge-Kutta method where the initial approximation uses fourth order Runge-Kutta and the error estimate uses a fifth order Runge-Kutta method. The error estimate is then used to adapt the time step [3]. We use an explicit rather than implicit time-stepping method as the integral component $\mathrm{K}(\mathrm{u})$ results in a nonlinear matrix that becomes computationally expensive to solve. For this scheme we derive the stability condition [2] as

$$
\max \left(\left|\frac{\mathrm{D}}{\Delta \mathrm{x}_{i}^{2}}+\frac{\mathrm{K}\left(\mathrm{U}_{\mathrm{i}}\right)}{\Delta \mathrm{x}_{\mathrm{i}}}\right|\right) \Delta \mathrm{t} \leqslant 4 .
$$

\subsection{Optimisation}

We investigate two spatial optimisation schemes. As the solution tends towards aggregations we tested a volume based refinement method. For this method the mean volume of the grid cells is $\mathrm{m}=\frac{1}{\mathrm{~N}} \sum_{i=0}^{N} \Delta \mathrm{x}_{i} \mathrm{U}_{i}$, and for some constants $\alpha$ and $\beta$, we refine if

$$
\Delta x_{i} u_{i}>\alpha m
$$


and coarsen if

$$
\Delta x_{i} u_{i}+\Delta x_{i+1} u_{i+1}<\beta m .
$$

The second refinement scheme is based on a refinement method presented by Van Loan [3, §3.1.4]. In this scheme we refine if the absolute difference between an average density of two cells and the cell in between is more than some constant,

$$
\left|\frac{\mathrm{U}_{i}+\mathrm{U}_{i+2}}{2}-\mathrm{U}_{i+1}\right|>\alpha,
$$

and coarsen if the absolute value of the gradient between two cells is less than a predefined value, that is,

$$
\left|\frac{u_{i+1}-u_{i}}{h_{i+\frac{1}{2}}}\right|<\beta .
$$

Refinement is achieved by splitting a cell into two cells with equal density and cell width. For coarsening, two cells are combined and the resulting cell has a density equal to the weighted average of the two combined cells, that is,

$$
\mathrm{u}_{\text {new }}=\frac{\mathrm{u}_{i} \Delta \mathrm{x}_{\mathrm{i}}+\mathrm{U}_{\mathrm{i}+1} \Delta \mathrm{x}_{\mathrm{i}+1}}{\Delta \mathrm{x}_{\text {new }}},
$$

for $x_{\text {new }}$ the width of the new grid cell.

\section{Results}

We ran a series of simulations on two population densities $u$ and $v$, given by

$$
\begin{aligned}
& \frac{\partial u}{\partial t}=D_{u} \frac{\partial^{2} u}{\partial x^{2}}-\frac{\partial}{\partial x}\left[u K_{u}(u, v)\right] \\
& \frac{\partial v}{\partial t}=D_{v} \frac{\partial^{2} v}{\partial x^{2}}-\frac{\partial}{\partial x}\left[v K_{v}(u, v)\right],
\end{aligned}
$$


where

$$
\begin{aligned}
\mathrm{K}_{u}(u, v)= & S_{u \mathfrak{u}} \int_{-1}^{1} \max [u(1-u-v), 0] \operatorname{sign}\left(x_{0}\right) d x_{0} \\
& +S_{u v} \int_{-1}^{1} \max [v(1-u-v), 0] \operatorname{sign}\left(x_{0}\right) d x_{0}, \\
K_{v}(u, v)= & S_{v v} \int_{-1}^{1} \max [v(1-u-v), 0] \operatorname{sign}\left(x_{0}\right) d x_{0} \\
& +S_{v u} \int_{-1}^{1} \max [u(1-u-v), 0] \operatorname{sign}\left(x_{0}\right) d x_{0} .
\end{aligned}
$$

To test the validity of our refinement methods we ran simulations corresponding to those presented by Armstrong et al. [1]. On comparing our results with those of Armstrong et al. we observe qualitatively similar behaviour. All simulations are run with periodic boundary conditions, that is $\mathfrak{u}(t, L)=\mathfrak{u}(t, 0)$. The spatial domain is $x=[0,20]$, the time domain is $t=[0,500]$, and initial conditions are $u(0, x)=0.2+\mu, v(0, x)=0.2+\mu$ where $\mu$ is some uniformly distributed noise, $\mu \sim \mathcal{U}(0,0.01)$. For simplicity, in all cases $S_{v \mathfrak{u}}=S_{u v}=C$ for constant $\mathrm{C}$.

For the gradient based refinement method $\alpha=0.01$ and $\beta=0.0005$, the minimum grid spacing is $\Delta x_{\min }=0.025$, and the maximum grid spacing is $\Delta x_{\max }=0.2$. For the reference simulation the maximally refined grid is $\Delta x=0.025$. Finally, as the volume based method performed poorly in terms of accuracy, in the interest of brevity the corresponding results are not presented here.

Figure 1 shows the results corresponding to cellular mixing when the two cell types evenly occupy the same space. To simulate this behaviour $\mathrm{D}=1$, $S_{u}=25, S_{v}=7.5$ and $C=22.5$. Figure 2 shows the engulfment results when one cell type completely engulfs the other. To simulate engulfment $\mathrm{D}=0.1, \mathrm{~S}_{\mathfrak{u}}=25, \mathrm{~S}_{v}=2.5$ and $\mathrm{C}=5$. Figure 3 shows the partial engulfment results in which one cell type partially engulfs the other. To simulate this $\mathrm{D}=1, \mathrm{~S}_{\mathfrak{u}}=25, \mathrm{~S}_{v}=25$ and $\mathrm{C}=12.5$. Figure 4 shows the 

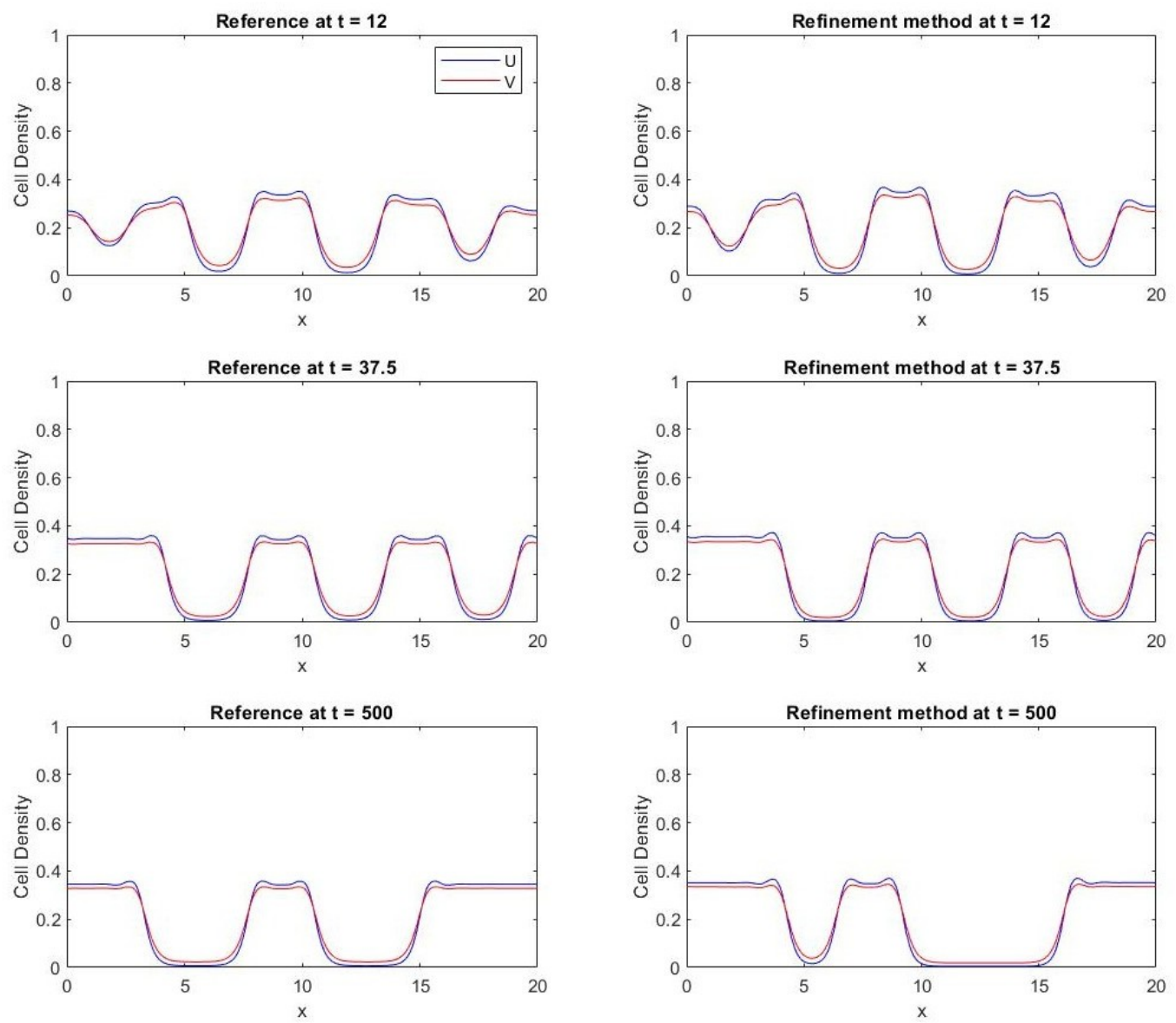

Figure 1: For cell mixing we used $D=1, S_{\mathfrak{u}}=25, S_{v}=7.5$ and $C=$ 22.5. The qualitative behaviour of the maximally refined grid reference simulation (left) and refinement method (right) look is similar. The differences in the two simulations may be due to a limitation with the finite volume method, as discussed in Section 4. 

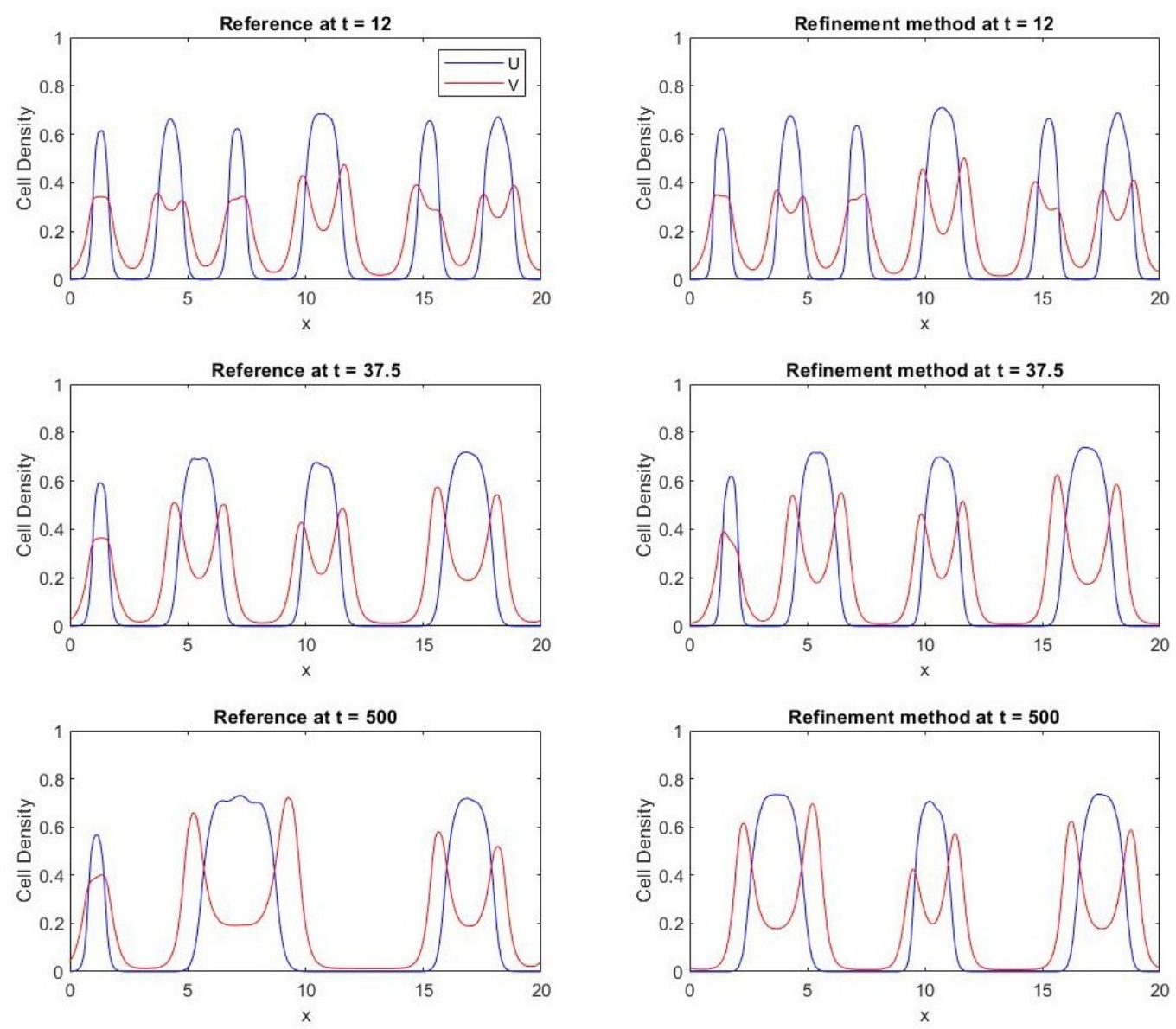

Figure 2: For full engulfment we used $D=0.1$ and $S_{u}=25, S_{v}=2.5$, $\mathrm{C}=5$. The qualitative behaviour of the maximally refined grid reference simulation (left) and refinement method (right) is very similar. The differences in the two simulations may be due to a limitation with the finite volume method, as discussed in Section 4. 

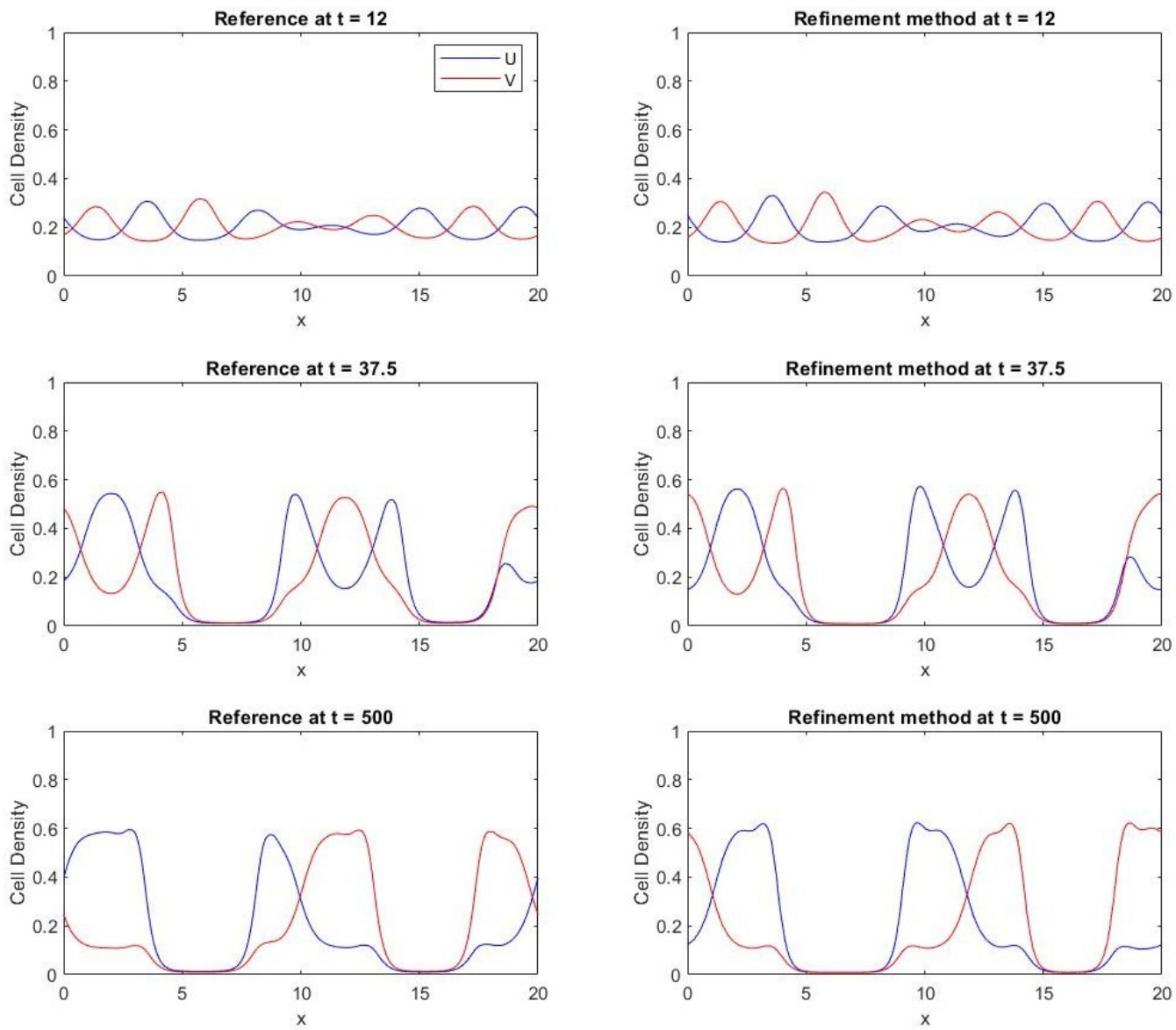

Figure 3: For partial engulfment we used $\mathrm{D}=1$ and $\mathrm{S}_{\mathfrak{u}}=25, \mathrm{~S}_{v}=25$, $\mathrm{C}=12.5$. The qualitative behaviour of the maximally refined grid reference simulation (left) and refinement method (right) is very similar. The differences in the two simulations may be due to a limitation with the finite volume method, as discussed in Section 4. 

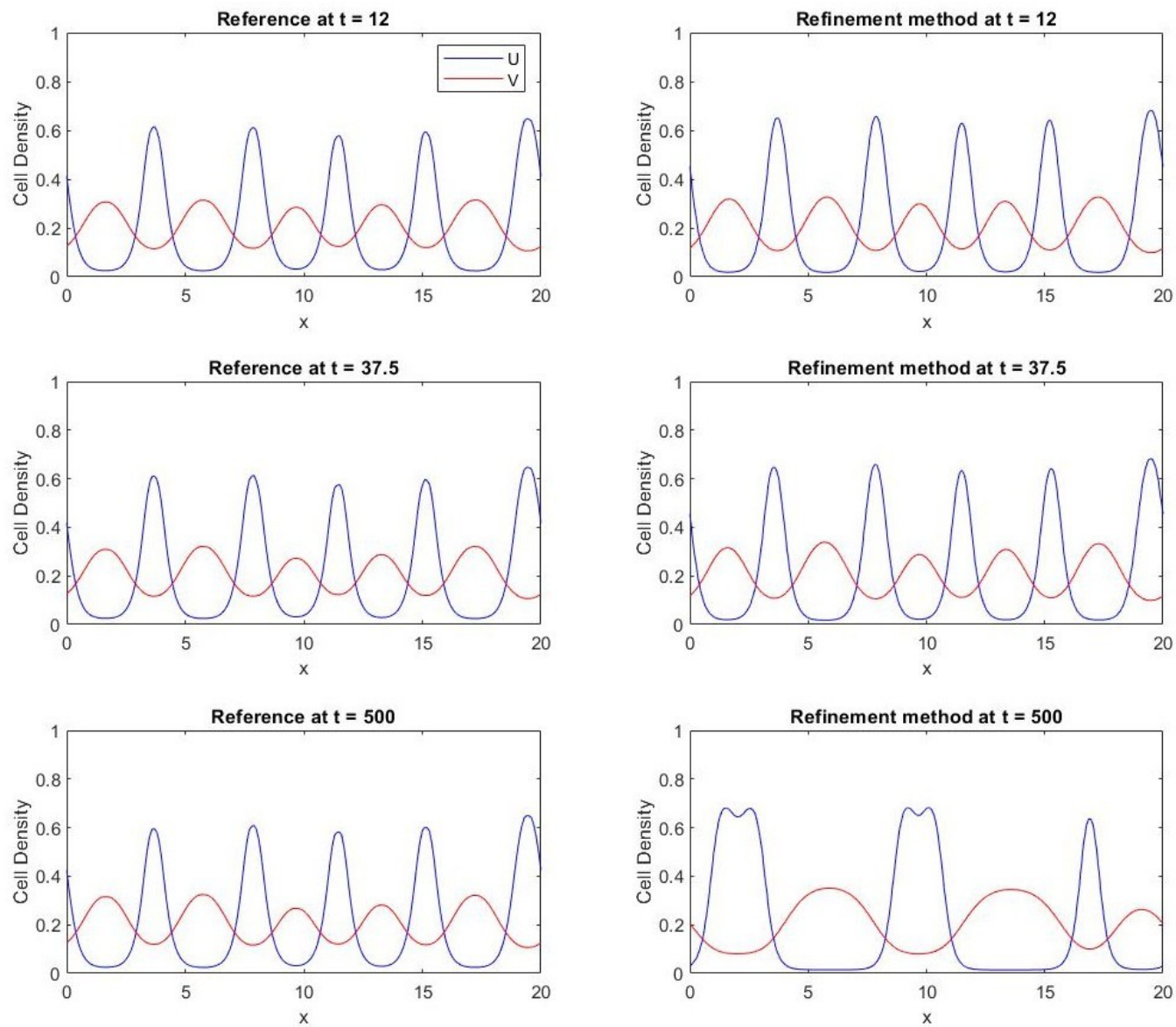

Figure 4: Cell Sorting, the following values were used $D=1, S_{\mathfrak{u}}=25$, $\mathrm{S}_{v}=7.5, \mathrm{C}=0$. The qualitative behaviour of the maximally refined grid reference simulation (left) and refinement method (right) is very similar. The differences in the two simulations may be due to a limitation with the finite volume method, as discussed in Section 4. 
Table 2: Computation time comparison between the gradient based refinement method (GBR) and a maximally refined grid (MRG), it can be seen that the refinement method vastly improves computation times. All computations were performed on the same computer under the same running conditions.

\begin{tabular}{ll|ll|ll|ll}
\multicolumn{2}{l|}{ Mixing } & \multicolumn{2}{|l|}{ Engulfment } & \multicolumn{2}{l|}{ Partial Engulfment } & \multicolumn{2}{l}{ Sorting } \\
\hline GBR & MRG & GBR & MRG & GBR & MRG & GBR & MRG \\
1836 & 108798 & 317 & 12135 & 2047 & 109613 & 2934 & 112894
\end{tabular}

sorting results whereby the two cell populations sort into their different types. To simulate sorting $D=1, S_{\mathfrak{u}}=25, S_{v}=7.5$ and $C=0$. Figures $1-4$ show qualitative agreement between the maximally refined grid simulations and the gradient based refinement method, and these simulations are also qualitatively similar to the original results of Armstrong et al. [1, Fig. 9]. Section 4 considers the quantitative differences between these three results. Finally, the computation times of the reference maximally refined grid and the gradient based refinement method are presented in Table 2.

\section{Conclusion}

We developed an adaptive spacial scheme for a partial-integro differential equation that considerably reduces computational costs and maintains qualitative behaviour. However, there are differences in the quasi-steady states that might be introduced by the relationship between grid spacing, the integral component of the equation, and the averaging step within the finite volume method. In other words, after each time step the value of $u_{i}$ is averaged over the cell $i$; if the cell spacing is too large this may change the integral component of the equation $\mathrm{K}(\mathrm{u})$ in cells within the sensing radius $\mathrm{R}$, thus causing movement that otherwise would not occur. To rectify this more time needs to be spent deciding on minimum and maximum grid spacings, when to perform the refinement, as well as better characterisation of the errors. 


\section{References}

[1] N. J. Armstrong, K. J. Painter, and J. A. Sherratt. "A continuum approach to modelling cell-cell adhesion". In: J. Theor. Biol. 243 (2006), pp. 98-113. DOI: 10.1016/j.jtbi.2006.05.030 (cit. on pp. C188, C189, C193, C198).

[2] R. J. LeVeque. Finite volume methods for hyperbolic problems. Cambridge Texts in Applied Mathematics. Cambridge University Press, 2002. DOI: 10.1017/CB09780511791253 (cit. on pp. C190, C191).

[3] C. F. Van Loan. Introduction to scientific computing: A matrix vector approach using MATLAB. MATLAB Curriculum. Prentise Hall, 1997. Chap. 3, pp. 101-104 (cit. on pp. C191, C192).

[4] J. A. Sheratt, S. A. Gourley, N. J. Armstrong, and K. J. Painter. "Boundedness of solutions of a non-local reaction-diffusion model for adhesion in cell aggregation and cancer invasion". In: Eur. J. Appl. Math. 20.1 (2009), pp. 123-144. DOI: 10.1017/S0956792508007742 (cit. on p. C189).

[5] M. S. Steinberg. "Mechanism of tissue reconstruction by dissociated cells, II: Time-course of events". In: Science 137.3532 (1962), pp. 762-763. DOI: 10.1126/science.137.3532.762 (cit. on p. C188).

[6] M. S. Steinberg. "On the mechanism of tissue reconstruction by dissociated cells, I. Population kinetics, differential adhesiveness and the absence of directed migration". In: P. Natl Acad. Sci. USA 48.9 (1962), pp. 1577-1582. DOI: 10.1073/pnas.48.9.1577 (cit. on p. C188).

[7] M. S. Steinberg. "On the mechanism of tissue reconstruction by dissociated cells, III. Free energy relations and the reorganisation of fused, heteronomic tissue fragments". In: P. Natl Acad. Sci. USA 48.10 (1962), pp. 1769-1776. DOI: 10.1073/pnas.48.10.1769 (cit. on p. C188). 
[8] P. L. Townes and J. Holtfreter. "Directed movements and selective adhesion of embryonic amphibian cells". In: J. Exp. Zool. 128.1 (1955), pp. 53-120. DOI: 10.1002/jez.1401280105 (cit. on p. C188).

\section{Author addresses}

1. F. Georgiou, School of Mathematical and Physical Sciences, University of Newcastle mailto:fillipe.georgiou@uon.edu.au orcid:0000-0003-4588-5319

2. B. P. Lamichhane, School of Mathematical and Physical Sciences, University of Newcastle

3. N. Thamwattana, School of Mathematical and Physical Sciences, University of Newcastle 